See discussions, stats, and author profiles for this publication at: https://www.researchgate.net/publication/303514996

\title{
Contrasting Cases: The Lotka-Volterra Model Times Three
}

Chapter · May 2016

DOI: $10.1007 / 978-3-319-30229-4 \_8$

2 authors:

Tarja Knuuttila

University of Vienna

94 PUBLICATIONS 1,361 CITATIONS

SEE PROFILE
Andrea Loettgers

University of Vienna

34 PUBLICATIONS 283 CITATIONS

SEE PROFILE

Some of the authors of this publication are also working on these related projects:

Prect Ontologisch Status of Organizationsl Principles in Biology View project

Possible Life: The Philosophical Significance of Extending Biology (ERC) View project 


\title{
Chapter 1 \\ Contrasting Cases: \\ The Lotka-Volterra Model Times Three
}

Tarja Knuuttila and Andrea Loettgers

\begin{abstract}
How do philosophers of science make use of historical case studies? Are their accounts of historical cases purpose-built and lacking in evidential strength as a result of putting forth and discussing philosophical positions? We will study these questions through the examination of three different philosophical case studies. All of them focus on modeling and on Vito Volterra, contrasting his work to that of other theoreticians. We argue that the worries concerning the evidential role of historical case studies in philosophy are partially unfounded, and the evidential and hermeneutical roles of case studies need not be played against each other. In philosophy of science, case studies are often tied to conceptual and theoretical analysis and development, rendering their evidential and theoretic/hermeneutic roles intertwined. Moreover, the problems of resituating or generalizing local knowledge are not specific to philosophy of science but commonplace in many scientific practices-which show similarities to the actual use of historical case studies by philosophers of science.
\end{abstract}

\subsection{Introduction}

Philosophers of science make frequent use of case studies, and the use of case studies has become even more prevalent in recent years with the more marked practiceorientation of even mainstream philosophy of science. Yet the case study methodology is rarely discussed by philosophers of science, and even more rarely is their own use of case studies reflected on. Some philosophical reflections on case studies (e.g. by Shrader-Frechette and McCoy (1994), Morgan (2012), Morgan (2014),

Tarja Knuuttila

Department of Philosophy University of South Carolina, USA, e-mail: tarja.knuuttila@helsinki.fi

Andrea Loettgers

Center for Space and Habitability, University of Bern, Switzerland, and Department of Philosophy, University of Geneva, Switzerland; e-mail: andrea.loettgers@unige.ch 
Ankeny (2012)), discuss the use of cases in scientific discourses but not as vehicles of philosophical theorizing. The lack of reflection among philosophers concerning their own use of case studies seems curious, since, on the face of it, the philosophical use of cases might seem problematical-unless they were understood as a mere means of illustration. The issue, of course, concerns generalization. Philosophical reflection often moves on a general conceptual level, and the question is how a single case, or any limited number of cases, for that matter, is going to give us general insights. In other words, is there a gap between specific historical cases and general philosophical theorizing?

Pitt (2001) thinks that this is indeed the case: according to his analysis, the use of historical cases as evidence for philosophical theorizing is highly problematical. In particular, their use is subject to the following dilemma. On the one hand, if the cases were used to back up a general philosophical claim, the question is how one, or a few, episodes of science can really establish it, and, furthermore, to what extent they have been fabricated in order to fit the philosophical thesis in question. On the other hand, if one starts from the case study and attempts to work one's way up to interesting philosophical conclusions, it is far from clear how this is supposed to be happening. Pitt's rather skeptical conclusions concerning the possible role of historical case studies in philosophy has generated a debate with some of counterarguments and qualifications. Pitt concludes: "even very good case studies do no philosophical work. They are at best heuristics" (Pitt (2001), p. 373). In response to Pitt, Burian (2001, p. 387) argued that Pitt's “ 'dilemma is a false dilemma.' " $\mathrm{He}$ demonstrates with reference to some examples drawn from the history of molecular biology that case studies help to shed light on such styles of scientific work and modes of argumentation that had not, so far, received due recognition in standard philosophical analyses. According to Burian, Pitt's dilemma only applies if we take the doubtful view of philosophy of science as aiming at the discovery of a universal or objective scientific method. He sees more fruitful philosophical work done in particular contexts, offering only limited, fallible generalizations-yet having also transformative power in regard to our view of science.

Writing a decade later, in reviewing the history of history and philosophy of science (HPS) and the confrontations between the two disciplines, Schickore (2011) takes up the argument between Burian and Pitt. She agrees with Pitt's conclusion that it is unjustified to generalize on the basis of one or a few episodes to wholesale claims concerning science-adding that the same applies for attempts to discard any such claims drawing only on a case or two (a genre that has been particularly vigorous in science and technology studies). What she does not believe in are attempts at rescuing the case study methodology by using sets of longitudinal or comparative case studies - an approach she attributes to Burian. Instead, Schickore thinks that there is something fundamentally wrong in approaching philosophical analysis in terms of the practice of natural science, which seeks to confront the theory with evidence. According to such "confrontation model", philosophy of science is developing a theory of science and the role of history of science is to provide data for its confirmation or falsification. In contrast to this confrontation model, Schickore advances a hermeneutical account that generates understanding of how scientific 
concepts, norms, and practices have developed. The hermeneutic circle of Schickore's proposal contains both historical and philosophical insights and allows the modification of concepts through the work on historical cases.

Recently, Kinzel (2015) has portrayed this discussion by distinguishing between the evidential and hermeneutic approaches. ${ }^{1}$ She points out, rightly we think, that the confrontation model does not work in the natural sciences either, and so argues for the evidential role of historical case studies. They are, according to her, capable of providing some evidential support for philosophical theorizing, provided that their theory-laden narrative and constructed nature are properly understood. The above short description of the discussion spawned by Pitt's skepticism concerning the philosophical use of historical case studies shows that the discussion has tended to revolve around the issues concerning generality versus locality of the claims supported by case studies, and the hermeneutic versus evidential functions of historical cases. In particular, it seems that all the participants of the debate (except perhaps Burian) take it that the hermeneutic/narrative/theory-laden nature of historical case studies limits their evidential role. ${ }^{2}$ But does this need to be the case? Is it possible to conceive of historical (and empirical) case studies in a way that does not see the interpretative, theory-laden nature of historical case studies as a limitation for their evidential role for philosophical reasoning? In short, is it possible to conceive of a particular philosophical way of using historical case studies that combines their evidential-cum-theoretical nature? And how would such an account stand in terms of the question concerning the general versus local nature of case-based reasoning?

In what follows, we will approach these questions through the examination of three different philosophical case studies, one of which was authored by us. As philosophers of science, having studied and presented several case studies (e.g. Knuuttila (2006, 2013); Loettgers (2007); Knuuttila and Loettgers (2013, 2014)), we will engage in this article in self-reflection of our own method presenting one of our case studies (Knuuttila and Loettgers, 2015). Our endeavor is motivated by the realization that the discussion on the use of historical case studies within philosophy of science so far has largely proceeded on a rather general level. Consequently, a more case-based investigation of the philosophical use of historical cases may seem in order.

The three different case studies we will consider focus on modeling and on the work of the Italian physicist and mathematician Vito Volterra-they all take his version of the Lotka-Volterra model as a point of departure. The model is a staple of philosophical discussion about mathematical modeling. What binds the three case studies together is the fact that both our own discussion (Knuuttila and Loettgers, 2015) and that of Scholl and Räz (2013) were written in part as responses to Michael Weinberg's (2007) influential “ 'Who is a Modeler?' " In this article, Weisberg contrasts Volterra's version of the Lotka-Volterra to Mendeleev's periodic table claiming that of the two scientists only Volterra was a modeler. Weisberg then

\footnotetext{
${ }^{1}$ Kinzel gives also Chang (2012) as an example of a proponent of a hermeneutic approach.

${ }^{2}$ Some vestiges of the confrontation model seem to be at play here, even in the rejection of it.
} 
uses the contrast between Volterra's and Mendeleev's styles of theorizing to support his claim that modeling is a distinct form of theorizing.

What is especially interesting about the three studies on Volterra's modeling is that all of them contrasted his work to that of another theoretician to deliver their philosophical points. The contrasts used were different, however. Scholl and Räz (2013) contrasted Volterra's modeling to Darwin's theory of the formation and distribution of coral atolls in the Pacific Ocean (also discussed by Weisberg (2007)). In our work, we contrasted Volterra's version of the Lotka-Volterra model to Lotka's version of it (Knuuttila and Loettgers, 2013). We suggested that the contrast between Volterra and Lotka provides interesting material for evaluating Weisberg's claims concerning modeling since Weisberg only considered Volterra's construction of the Lotka-Volterra model. Our case studies showed that although Volterra and Lotka presented models that, from a formal point of view, looked identical, they nevertheless followed different kinds of modeling strategies (Knuuttila and Loettgers, 2015).

In what follows, we will first discuss Michael Weisberg's contrasting cases, turning then to Scholl and Räz's (2013) article that makes use of a different contrast in arguing against Weisberg's position. Our own case study follows. In the discussion and concluding chapter, we will contrast and compare the three different case studies with each other, asking what kind of philosophical insight they offer, and how they do it. In particular, we will suggest that the worries concerning the evidential role of case studies, and their contextualization, are partially based on an inadequate understanding of how historical case studies are used in actual philosophical practice. As philosophical discourses are often tied to conceptual analysis and development, the evidential and hermeneutic roles are intertwined in case-based reasoning. Cases are often studied in order to advance a philosophical argument, and in such use case studies provide both evidence and interpretative resources for exploring and developing philosophical concepts and theories. In this work, various ways of expanding the depth and coverage of the case-based argumentation may be used, one of which is making use of the vehicle of contrasting different scientific cases.

\subsection{Modeling as indirect representation}

In his “ 'Who is a Modeler?' " Michael Weisberg (2007) argues that many standard philosophical accounts fail to distinguish between different forms of theorizing. What he is interested in is articulating modeling as a distinct theoretical practice. According to him, the goals, procedures, and representations employed by modelers and other kinds of theorists differ. In particular, he distinguishes between two types of theorizing: modeling and abstract direct representation. Modelers, according to Weisberg, study real-world phenomena through the detour of creating hypothetical simplified entities, models. That is, they practice the art of indirect representation. In contrast, the theorists practicing abstract direct representation strive to represent the data or real-world phenomena directly. 
The central philosophical thesis of " 'Who is a Modeler?' "revolves, then, around the notion of indirect representation and the peculiar way models relate to realworld phenomena. Models form a class of theoretical representations that are not constructed by representing the real target systems. The consideration of the latter first enters the process of modeling at a later stage that runs counter to the traditional representational idea of models as inherently models of some real target systems. ${ }^{3}$ The claim that model construction happens before the possible real target systems are being considered challenges the traditional understanding of models as representations of some real-world target systems. Much of the scientific discussion has (explicitly or implicitly) taken models as prototypical scientific representations and proceeded to analyze the notion of representation through modeling (see, e.g., Bailer-Jones (2009); Contessa (2007); da Costa and French (2000); French and Ladyman (1999); Frigg (2010); Giere (2004); Morgan and Morrison (1999); Maeki (2009); Suárez (2008)). Weisberg departs from such assumptions in pointing out the diversity of ways in which theoretical representations may be built, and related to real world systems (see also Weisberg (2013)).

In making the distinction between modeling and abstract direct representation, Weisberg redirects the focus from models to the activity of modeling. He proceeds in three stages. Firstly, a model is being constructed, after which, secondly, the modeler refines, analyses, and articulates its properties and dynamics. Only at the third stage, the relationship between the model and any real-world target system is assessed, if such an assessment is deemed necessary. Often modelers are predominantly interested in studying model systems themselves, and so the relationship between model and any real-world target may be left implicit at best. This characteristic feature of contemporary modeling practice tends to go unnoticed if models are understood in the traditional representational fashion.

Interestingly, after characterizing abstract direct representation and modeling in a preliminary fashion, Weisberg proceeds to analyze this distinction in a more fine-grained manner making use of two cases. He contrasts Vito Volterra's modeling style of theorizing from abstract direct representation as exhibited by Dimitri Mendeleev's periodic table. In his argument, Weisberg makes only use of Volterra's articles published in 1926 in Italian and English (Volterra, 1926a,b). According to Weisberg, Volterra studied post-World War I fish populations in the Adriatic Sea by 'imagining a simple biological system composed of one population of predators and one population of prey' (Weisberg, 2007, p. 208). He attributed to this hypothetical system only a few properties writing down a couple of differential equations to describe their mutual dynamics. The word 'imagining' used by Weisberg captures the difference between the procedures of direct and indirect representation. He underlines that Volterra did not arrive at these model populations by abstracting away from properties of real fish, but rather constructed them by stipulating certain of their properties (Weisberg, 2007, p. 210). In contrast to Volterra, Weisberg claims, Mendeleev built his Periodic Table through abstractions from data in order to identify some central factors of chemical behavior. Thus, in contrast to modelers con-

\footnotetext{
${ }^{3}$ In recent discussion on modeling, the idea of models as fictions has been entertained by several authors, e.g. Suárez (2008).
} 
structing hypothetical systems, he was trying to 'represent trends in real chemical reactivity, and not trends in a model system' (Weisberg, 2007, p. 215, footnote 3 ). ${ }^{4}$

Both abstract direct representation and indirect representations abstract, approximate, select, and idealize, so the difference between the two does not hang on these procedures of scientific representation. What distinguishes modeling from abstract direct representation is that it proceeds by describing another simpler, hypothetical system. Consequently, models should be considered independent objects in the sense of being independent from some determinable real target systems. ${ }^{5}$ This implies also an important difference on how one gains knowledge via modeling and abstract direct representation. Namely, Weisberg claims that in abstract direct representation 'anything the theorist discovers in her analysis of the representation is a discovery about the phenomenon itself, assuming that it was represented properly. There is no extra stage where the theorist must coordinate the model to a real phenomenon'-as is the case with modeling (Weisberg, 2007, pp. 226-227). Although it seems that Weisberg exaggerates the extent to which abstract direct representation can allow the direct study of the phenomenon, yet such difference may explain why the discussion on scientific representation has focused on modeling. Models seem to provide the hard case that the various accounts of scientific representation are designed to address.

As already mentioned, Weisberg's account pays attention to the fact that models are often studied quite apart from any representational relationships that they might have to real-world systems. Moreover, many scientific models are far too simple to be considered as models of some actual target systems, although they may bear similarities to them. Secondly, scientists also study models of phenomena that are not known to exist. If it is better understood why these phenomena do not exist, one has also gained some understanding of the phenomena that do exist. In both of the aforementioned situations, Weisberg claims that 'it is clear that the model and only the model is the object of study' (Weisberg, 2007, p. 223).

While Weisberg's thesis of modeling as indirect representation thus brings to the fore some aspects of modeling largely neglected by the philosophical discussion so far, the account seems lacking in some crucial respects. This becomes visible if we examine how Weisberg thinks a modeler can be recognized: 'To judge whether or not a particular theorist is a modeler,' argues Weisberg, '[w]e will actually need to know something about how the theory was developed and how the modeler set about trying to represent the world' (Weisberg, 2007, p. 222). Let us consider, then, what Weisberg says about how Volterra went about developing his version of the Lotka-Volterra model:

\footnotetext{
${ }^{4}$ Godfrey-Smith (2006) likewise distinguishes between indirect representation and abstract direct representation - and invokes examples in trying to account for the difference between the two strategies of theorizing. Godfrey-Smith studies two influential books on evolutionary theory: Leo Buss's The Evolution of Individuality (1987) and Maynard Smith and Szathmáry's The Major Transitions in Evolution (1995). Buss examines the 'actual relations between cellular reproduction and whole-organism reproduction in known organisms' (Godfrey-Smith, 2006, p. 731), while Maynard Smith and Szathmáry put forth 'idealized, schematic causal mechanisms.'

5 On models as independent or autonomous entities, see also Morgan and Morrison (1999) and Knuuttila (2005).
} 
Volterra began his investigation of Adriatic fish not by looking directly at these fish or even the statistics gathered from the fish markets, but by constructing a model. This is characteristic of the first stage of modeling. He imagined a population of predators and a population of prey, each with only two properties. Setting this idea to paper, he wrote down equations specifying the model that he had imagined. (Weisberg, 2007, p. 222, emphasis added).

We find this a gross simplification of actual modeling practice and its reliance on the already established computational methods and representational tools. In our case study, we will study how Volterra and Lotka constructed their respective models. As we will argue, Volterra does not qualify as a prime example of a modeler, since he pursued the essential or sufficient components of the real predator-prey system. Although what he eventually accomplished suits Weisberg's claims about modeling, his original intentions were nonetheless different. Lotka provides a better example of a modeler, but for reasons that are not discussed by Weisberg. Lotka started from a systems theoretical perspective, developing a general model template, which he applied to the analysis of biological and chemical systems. This kind of approach is becoming prevalent in modeling complex systems. It does not start from imagining simplified hypothetical systems (still somehow connected to some particular real-world systems) but from applying cross-disciplinary computational templates to various subject matters (cf. Humphreys $(2002,2004)$ ). Before going into our case studies, we will discuss the critique of the thesis of indirect representation by Scholl and Räz (2013). They approach modeling in terms of 'insufficient epistemic access' and use as contrast cases Volterra and Darwin, a comparison already made by Weisberg (2007). However, Scholl and Räz are mainly referring to Volterra's much later work, co-authored with D'Ancona (Volterra and D'Ancona, 1935), whereas Weisberg relies exclusively on Volterra's original articles from 1926 (Volterra (1926a,b)). On our view, this may partially explain their different interpretations of Volterra.

\subsection{Modeling is not about indirect representation}

Although Weisberg launches the contrast between abstract direct representation and indirect representation mainly in terms of the contrast between Volterra and Mendeleev, later in his article he also discusses Darwin's theory of the origin and distribution of the coral reefs as an example of abstract direct representation (Weisberg, 2007, pp. 227-228). Scholl and Räz (2013) focus on the contrast between Volterra's and Darwin's causal reasoning in their critique of Weisberg's thesis of indirect representation. Despite their critique, Scholl and Räz's interpretation of Volterra is also very much in line with what Weisberg claims. They point out that according to Volterra and D'Ancona their "deductive approach" does not attempt to " 'extract ecological laws directly from experimental data or observation.' " Instead, it proceeds on a " "constructive path" " through hypotheses about basic causal relationships, integrating them " 'into a system of interactions' " (Scholl and Räz, 2013, p. 120). Moreover, they adopt from Weisberg the distinction between dynam- 
ical and representational fidelity (see below). Where the two interpretations differ most, is how Weisberg, on the one hand, and Scholl and Räz, on the other hand, define modeling.

While Weisberg uses the contrast between Volterra's, and Mendeleev's (and Darwin's) approaches to elucidate further his notion of modeling as indirect representation, Scholl and Räz extract their notion of modeling from Volterra and D'Ancona (1935), arguing that Volterra and D'Ancona chose a modeling approach because direct methods were not available for the problem they were studying. What would have been the direct methods? ${ }^{6}$ Volterra and D'Ancona (1935) distinguish and discuss three different " "direct' " methods. Each of the methods has their own limitations when it comes to the studying of predator and prey dynamics. Firstly, there is the experimental method of studying individual causes in isolation in controlled conditions. Experiments on individual animals under laboratory conditions would allow for causal inference, but ecologists study interactions of entire populations of animals, making this approach deemed unsuitable by Volterra and D'Ancona. Consequently, the second alternative would be to overcome this limitation by transferring the method of causal inference to ecology by performing breeding experiments on entire populations.

In order to perform such controlled breeding experiments several requirements have to be fulfilled: A space whose dimension has to be proportional to the size of the animal is needed to run the experiment, and the length of the experiment has to comply with the life expectations of the animals and their breeding cycles. Finally, the environmental conditions would need to be controlled. Such experiments were actually performed by the Russian biologist G. F. Gause (1935) who used microorganisms instead of fish. Micro-organisms have two important advantages: they do not need a lot of space and have short generation times. Under controlled laboratory conditions, Gause was able to explore how the prey micro-organisms developed in isolation, and how the situation changed when the predator micro-organisms were added. As promising as these experiments seemed to be to Volterra and D'Ancona, they turned out not to be able to replicate the situation modeled by the Lotka-Volterra model.

Finally, the third possibility considered by Volterra and D'Ancona, field experiments, seemed infeasible because of the large number of uncontrollable factors interfering with the population dynamics and would require intensive effort.

Based on the problems, or outright impossibility, of direct methods, Volterra and D'Ancona then argue in favor for modeling, i.e. for their " 'deductive method." " This leads Scholl and Räz to conclude that it was precisely the "insufficient epistemic access" " that forced the two scientists to resign to a modeling approach. Such an approach is described by the two authors as follows: "We begin with hypotheses about basic causal relationships and integrate them into a system of interactions. Then we check whether the constructed system, the model, is applicable to the target system"' (Scholl and Räz, 2013, p. 120). This procedure of constructing and applying a hypothetical model is very similar to Weisberg's indirect modeling ap-

\footnotetext{
${ }^{6}$ We are here largely following the discussion by Scholl and Räz (2013).
} 
proach. However, they proceed to claim, in contrast to Weisberg, that Darwin faced the same problem of insufficient epistemic access in his attempt to causally explain the formation, origin, and distribution of coral reefs and atolls in the Pacific Ocean. And so he was, by their criteria, also a modeler: " 'Darwin's investigation has all the hallmarks of model-based science ... The subsidence of islands and the growth of corals occur over hundreds of thousands of years, distributed over the entire Pacific Ocean, and so we can have no hope of directly investigating the process' " (ibid., 127).

The starting point of Darwin's modeling process was an island surrounded by a fringing reef. According to his observations, corals prefer warm and shallow waters, surrounding volcano islands. In imagining the hypothetical model, Darwin proceeded from fringing reefs to barrier reefs. The formation of barrier reefs starts with an island that sinks down. As a natural consequence also the corals will submerge under the water and die. These dead corals provide the basis for new growing corals, which will be now further away from the island because the island got smaller by sinking down. If the island keeps subsiding the coral reef keeps growing on its own foundation up the point where the island is completely under the water and forms therefore a coral atoll. In such step-by-step, reasoning Darwin was able to 'render all the known forms of coral islands' (ibid., 128).

The main difference between Volterra and D'Ancona and Darwin is not due to the method of modeling, according to Scholl and Räz, but rather the fact that Darwin was able to provide an " "how actually" " model of the formation of coral reefs and islands in contrast to the " "how possibly" " models of Volterra and D'Ancona. Darwin's account was able to " 'mirror' "- - a not so fortunate choice of word by Scholl and Räz-the causal structures of the target system, due to his careful observation of the coral reef formation at its different stages. Scholl and Räz depict Darwin's quest from a " 'how possibly' " model, via empirical demonstration of the hypothesized mechanism to adducing empirical evidence " "in support of the claim that the model faithfully represents the actual causal processes responsible for the growth of coral atolls" " (ibid., 130). As a result, his model was representationally faithful instead of succeeding only to reproduce empirical phenomena. Such dynamical fidelity was what Volterra and D'Ancona were eventually only able to accomplish.

Although thus Weisberg as well as Scholl and Räz are largely in agreement on their analysis of Volterra, they end up presenting his modeling exercise in different terms. In what follows, we will argue, presenting yet another case study on Volterra, that their disagreement can be partially settled by paying attention to the fact that they make use of different writings of Volterra. Whereas Weisberg focuses on the papers in which Volterra published his version of the Lotka-Volterra model for the first time (Volterra, 1926a,b), Scholl and Räz discuss at length the work that appeared nearly a decade later representing a mature state of a research program inspired by the early papers of Volterra and D'Ancona (1935). Furthermore, using Alfred Lotka's very different design of the Lotka-Volterra model as a contrasting case, we draw attention to the actual tools of modeling that both Weisberg as well as Scholl and Räz have glossed over in their analysis. The differences between Volterra and D'Ancona, and Darwin are to a large degree due to the fact that the former were 
engaged in mathematical modeling. Such theoretical activity is largely dependent on mathematical tools and methods that are often interdisciplinary by their nature. ${ }^{7}$

\subsection{The design of the Lotka-Volterra model by Volterra}

Weisberg begins his story of the origin of the Lotka-Volterra model with the problem presented by Umberto D'Ancona (1896-1964) to the world-renowned mathematical physicist Vito Volterra (1860-1940) in 1925. D'Ancona, a marine biologist and Volterra's son-in-law, had made a statistical study of the Adriatic fisheries over the period 1905-1923. The data showed an unusual increase in predators during the final period of the First World War and immediately after, when fishing was hindered by the war. D'Ancona's aim was to get mathematical support for the thesis that cessation of fishing was favorable for predator fish. Thus Volterra set out to " 'mathematically explain' " D' Ancona's data on " 'temporal variations in the composition of species" "(Volterra, 1927, p. 68). He had no prior experience of fisheries, yet this problem sparked his longer-term research program on the inter-species dynamics that culminated in Leçons sur la théorie mathématique de la lutte pour la vie (Volterra, 1931) and Les associations biologiques au point de vue mathématique (Volterra and D'Ancona, 1935). However, the way Volterra went about modeling the predator-prey system can be traced further back in time.

\subsubsection{Volterra and the mathematization of biology and social sciences}

Already decades before the formulation of the Lotka-Volterra model, Volterra was interested in the mathematization of biology and social sciences. At the opening of the academic year at the University of Rome in 1901, he delivered an Inaugural Address entitled On the Attempts to Apply Mathematics to the Biological and Social Sciences (Volterra, 1901). In this talk, Volterra spoke in favor of translating " "natural phenomena into arithmetical or geometrical language" " and by doing so opening " 'a new avenue for mathematics' " within biology and social sciences. An important ingredient in this transformation process was provided by mechanics: biology and social sciences should be mathematized according to the example provided by mechanics. For Volterra it constituted " "together with geometry, if not the most brilliant then surely the most dependable and secure body of knowledge' " (ibid., p. 251).

On the other hand, physics at the beginning of the 20th century was ridden by the apparent failure of the mechanistic world-view. This led Volterra to remark that instead of the " illusions about giving a mechanical explanation of the universe" "

\footnotetext{
${ }^{7}$ Our case studies are based on our earlier work (Knuuttila and Loettgers, 2015).
} 
one should " 'more modestly, [be] satisfied by analogy, and especially mathematical analogy" " (Volterra, 1901, p. 255). He thought that a large part of mathematical physics would still be usable, especially differential equations. ${ }^{8}$

The application of mathematics to social science and biology involved, for Volterra, transforming qualitative elements into quantitative measurable elements, measuring the variations, idealizing and abstracting the systems and processes under investigation, representing them with differential calculus, and forming hypotheses in the same fashion as in mechanics. The goal of idealization and abstraction was to identify the 'fundamental parameters' governing the 'change in the corresponding variable elements of the phenomena' (ibid., p. 255). Volterra saw in economics a good example of a science modeled on mechanics:

The concept of Homo economicus, which has prompted so much discussion and provoked such enormous difficulty that there are still those who refuse to accept it, comes so naturally to our mechanist that he is surprised at the suspicions aroused by this abstract, schematic being. He sees in Homo economicus a concept similar to those that, by long habit, have become familiar to him. He is used to idealizing surfaces as frictionless, wires as inextensible, solids as undeformable, and to substituting perfect liquids and gases for the natural kind. Not only has he made a habit of all this, but he also knows the advantages of doing so. (Volterra, 1901, p. 252).

Yet it appears contradictory to transfer the modeling methods and concepts of mechanics to another entirely different areas of study and strive, simultaneously, to capture the fundamental factors behind the phenomena in question. Is there a reason to suppose that the mechanical approach works in such fields as biology, or social sciences, taking into account the complexity of the phenomena they study? As we will show in the next section, this was precisely the reason why Volterra had to resort to 'the method of hypothesis' in modeling biological associations.

\subsubsection{Volterra's method of hypothesis}

According to his methodological ideals, Volterra embarked on accounting for D'Ancona's statistical data by " 'isolating those factors one wishes to examine, assuming they act alone, and by neglecting others' " (Volterra, 1927, p. 67). First, he distinguished between " 'external' " and " 'internal' " causes. External causes were " 'periodic circumstances relating to the environment, as would be those, for example, which depend upon the changing of the seasons, which produce oscillations of an external character in the number of the individuals of the various species'" (Volterra, 1928, p. 5). Volterra was focusing on internal causes that had " "periods of their own which add their action to these external causes and would exist even if these were withdrawn" "(ibid.). However, this was just a starting point for him since already at the beginning he had a larger picture in mind. He went on to model

\footnotetext{
${ }^{8}$ Volterra had started his scientific career as a mathematician and had made important contributions to the theory of calculus. This work is summarized in Volterra's book Theory of Functionals and of Integral and Integro-Differential Equations (Volterra, 1930).
} 
more complicated cases, adding also some effects of the environment. The LotkaVolterra model was merely one of the basic models of biological associations with which Volterra referred to stable associations that " 'are established by many species which live in the same environment" " (Volterra, 1928, p. 4). In the paper in which he presents the Lotka-Volterra model for the first time (Volterra, 1926b, 1928), ${ }^{9}$ he begins from a consideration of one species alone and then adds other species. The first association he models is that between two species which contend for the same supply of food. After that he formulated the Lotka-Volterra model on two species, one of which feeds upon the other.

Although Volterra strove to separate external and internal causes, he admitted that they could be interrelated in a myriad of ways. Interacting species in a changing environment constitutes a problem of a much higher degree of complexity than the systems studied in classical mechanics. The mathematical methods and techniques of mechanics could not be applied off-hand to the study of the predator-prey dynamics. Even if the variations observed in populations living in the same environment showed some well-known characteristics observed in many mechanical systems, such as oscillatory behavior, it was unclear, which were the components of the system and in which ways they interacted. Consequently, Volterra faced the following dilemma: On the one hand, the complexity of the system had to be rendered manageable, enabling the use of certain mathematical tools. On the other hand, the available mathematical tools and methods exhibited a serious constraint on the kinds of structures and processes that could be studied. Volterra reflected on this situation in the following way:

But on the first appearance it would seem as though on account of its extreme complexity the question might not lend itself to a mathematical treatment, and that on the contrary mathematical methods, being too delicate, might emphasize some peculiarities and obscure some essentials of the question. To guard against this danger we must start from the hypotheses, even though they be rough and simple, and give some scheme for the phenomenon. (Volterra, 1928, p. 5, emphasis added)

Since he could not isolate internal causes from external causes, due to the complexity of the interactions between the components of the system of interest, he constructed a hypothetical system with the help of certain assumptions concerning them. Some of these assumptions were directly due to the application of differential calculus to the problem of predation (see also our discussion on the method of isolation in section 5.1). The central assumptions made in the construction process of the model were:

- The numbers of species increase or decrease in a continuous way, which makes them describable by means of differential equations.

- Birth takes place continuously and is not restricted to seasons. The birth-rate is proportional to the number of living individuals of the species. The same assumption is made for the death rate.

\footnotetext{
9 (Volterra, 1928) is a partial English translation of the Italian original (Volterra, 1926b); in the following, references are made to the 1928 translation.
} 
- Homogeneity of the individuals of each species, which neglects the variations of age and size.

Thus Volterra concentrated exclusively on the dynamics between predators and preys by formulating a simplified hypothetical system consisting solely of " "the intrinsic phenomena" "due to the voracity and fertility of the co-existing species' (Volterra, 1927, p. 68). This strategy of " "starting from the hypotheses" " allowed Volterra to apply well-known mathematical tools and methods to the study of biological associations.

\subsubsection{The construction of the Lotka-Volterra model by Volterra}

In deriving the Lotka-Volterra equations, Volterra started out from a situation in which each of the species is alone. In this situation, he assumed, the prey would grow exponentially and the predator in turn would decrease exponentially, because of missing food resources. Translated into the language of mathematics, the development of prey and predator populations is described by the following two differential equations describing the change in time $t$ of the prey $\left(N_{1}\right)$ and predator $\left(N_{2}\right)$ populations:

$$
\frac{d N_{1}}{d t}=\varepsilon_{1} N_{1}, \quad \frac{d N_{2}}{d t}=-\varepsilon_{2} N_{2},
$$

where $\varepsilon_{1}, \varepsilon_{2}>0$ are constants. Integration of the two differential equations leads to an exponential increase of the prey and an exponential decrease of the predator population, with $N_{i, 0}$ referring to the numbers of individuals at time 0 .

$$
N_{1}(t)=N_{1,0} e^{\varepsilon_{1} t}, \quad N_{2}(t)=N_{2,0} e^{-\varepsilon_{2} t} .
$$

Exponential growth or decrease is the simplest way of describing the development of a population in time. It does not take into account any environmental influences or the obvious fact that there is must be an upper limit of the population sustainable by the resources provided by the environment. To allow for the interaction between prey and predator populations, Volterra introduced a coupling term in each equation. The combined predator and prey system is described by the following set of differential equations:

$$
\begin{aligned}
& \frac{d N_{1}}{d t}=\left(\varepsilon_{1}-\gamma_{1} N_{2}\right) N_{1}, \\
& \frac{d N_{2}}{d t}=\left(-\varepsilon_{2}+\gamma_{2} N_{1}\right) N_{2} .
\end{aligned}
$$

The interaction between predator and prey is now described by the terms involving the product $N_{1} N_{2}$, which introduces a non-linearity into the system in addition to the coupling of the two differential equations. The proportionality constant $\gamma_{1}$ links 
the prey mortality to the number of prey and predators, and the constant $\gamma_{2}$ links the increase in predators to the number of prey and predators. One of the possible solutions to these coupled non-linear differential equations is an oscillation in the numbers of predator and prey. Concerning those oscillating solutions Volterra wrote:

From the analytical viewpoint, it is to be noted that the study of fluctuations or oscillations of the number of individuals of species living together, [...] falls outside the ordinary study of oscillations, because in these researches we had to deal generally with non-linear equations, whereas the classical study of the theory of oscillations involves linear equations. (Volterra, 1928, p. 23)

The mathematical analysis of the resulting equations gave Volterra some important results-including a solution to D'Ancona's problem concerning the relative abundance of predatory fish during the war years. Volterra summarized his results in what he called the 'three fundamental laws of the fluctuations of the two species living together' (Volterra, 1928, p. 20). The third law states that if an attempt were made to destroy the individuals of the predator and prey species uniformly and in proportion to their number, the average number of the prey would increase and the average number of the predator would decrease. ${ }^{10}$ As regards fisheries, this " 'law" " was anticipated already by E. Ray Lancaster (1884). ${ }^{11}$ Volterra himself quoted Darwin: " 'If not one head of game were shot during the next twenty years in England, and at the same time no vermin were destroyed, there would in all probability be less game than at present, although hundreds of thousands of game animals are now annually shot" " (Volterra, 1926a, p. 559); (Darwin, 1882, pp. 53-54). For Volterra his long-term research on " 'biological associations" " was a contribution to the Darwinian theory of struggle for existence (see Volterra (1931); Volterra and D'Ancona $(1935)) .^{12}$

To appreciate the importance of mechanical analogies in the construction of Volterra's model one can, firstly, consider the way he treated predation. He assumed that the increase and decrease of predator and prey populations (Eqs. 1.3, 1.4) are linear with respect to the product of $N_{1}$ and $N_{2}$, i.e. $\gamma_{1}$ and $\gamma_{2}$ are constants. To justify this assumption Volterra drew an analogy to mechanics by using the so-called " 'method of encounters' " according to which the number of collisions between the particles of two gases is proportional to the product of their densities. ${ }^{13}$ Thus Volterra assumed that the rate of predation upon the prey is proportional to the product of the numbers of the two species.

Secondly, in generalizing his account to take into consideration the different kinds of interactions and multiple species, Volterra utilized mechanical analogies in various ways (e.g. Volterra (1926a, 1927, 1931)). For instance, making use of the concept of friction in mechanics he made a distinction between two types of

\footnotetext{
${ }^{10}$ For this so-called Volterra principle, see Weisberg and Reisman (2008).

${ }^{11}$ Lankester suggested that to protect edible prey-fish their enemies should be destroyed in the same proportion as the adult prey fish were 'removed' (Lancaster, 1884, p. 416).

12 On Volterra's Darwinism, see Scudo (1992).

13 Volterra made use of the method of encounters also in his study of the demographic evolution of a single species: There he applied the method of encounters to mating.
} 
biological associations, conservative and dissipative ones (Volterra, 1926a, 1927). Conservative systems are analogous to frictionless systems in mechanics. In conservative associations, the oscillations produced by the interactions of the species remain constant like in the Lotka-Volterra model. In dissipative associations, the fluctuations of the species are damped due to the friction caused by the interaction between individuals of the same species (which takes into account the effects of a population's size on its own growth). These cases display a parallel to the cases of damped and undamped harmonic oscillator in mechanics. Although conservative associations have very appealing mathematical properties, Volterra thought that dissipative associations are more realistic approximations of the natural situation than the conservative ones (Volterra, 1928, p. 47).

The tension between applying the concepts and mathematical techniques suggested by classical mechanics and the aim to construct more realistic models marked Volterra's long research program on biological associations. He spent the rest of his life, more than a decade, formulating more elaborate models, taking into account different kinds of associations and situations, and making extensive use of modeling methods borrowed from mechanics. Already in his original 1926 article (Volterra, 1926b), he also considered the cases of any number of species which either contended for the same food or some of which fed upon the others. One year after the publication of the original Italian article, Volterra (1927) also introduced integrodifferential equations in an attempt to take into account the delayed effects of feeding on reproduction. ${ }^{14}$ Finally, in a group of papers published in 1936 and 1937, Volterra made use of the calculus of variations in an attempt to provide a synthesis of his theory of biological associations along the lines of analytical mechanics. It is worth citing at length his explanation of this agenda:

The second part [of Principes de biologie mathématique] begins with a conservation of demographic energy, according to which there are two sorts of energy, one actual and one potential, which transform mutually the one into the other. The principle is the analogue of the principle of conservation of mechanical energy. It is followed by the enunciation of the three laws relating to the biological fluctuations, the experimental verification of which has been investigated by several naturalists. The success, which has attended their efforts is well known.

Everybody knows the importance of Hamilton's principle in mechanics and in all the domains of physical science. An analogous variation principle can be found in biology, and from it one can deduce the fluctuation equations in the canonical Hamiltonian form and also in the form of a Jacobian partial differential equation. [...]

Hamilton's principle leads to the principle of least action (Maupertuis). There exists also in biology a closely related principle, which may be called the principle of least vital action. Its analytical form is such that it requires the existence of a true minimum, a state of affairs which does not always hold good in the analogous case in mechanics. (Volterra, 1937b, p. 35$)^{15}$.

The reference to the experimental verification in the quotation above is important. Being faithful to his earlier methodological pronouncements, Volterra was also

\footnotetext{
14 Today Volterra is mostly known for the Lotka-Volterra equation. For a discussion on how Volterra's various models anticipated several theoretical advances in theoretical ecology, see Scudo (1971).

${ }^{15}$ A partial English translation of this paper can be found in (Scudo and Ziegler, 1978).
} 
interested in testing his theories on empirical data, although he typically kept the mathematical, technical treatments and the empirical accounts separate from each other. ${ }^{16}$ Apart from his collaboration with D'Ancona, he was also engaged in active correspondence with other biologists and scientists that served as an attempt for him to verify his theoretical findings empirically (see Israel and Gasca (2002)). ${ }^{17}$ Volterra rejected the idea of formulating mathematical models that could not be tested empirically and he insisted that all the variables introduced in the mathematical formalizations should be measurable. This eventually led him into a disagreement with D'Ancona, who was skeptical of Volterra's quest for empirical validation. He argued that Volterra's models were rather interesting theoretical working hypotheses able to stand on their own (see Israel $(1991,1993)$ ). Volterra's preference of grounding hypotheses in empirical research is displayed also by his reply to Alfred Lotka (Israel and Gasca, 2002). Lotka had claimed priority for the LotkaVolterra model on the basis of his Elements of Physical Biology (1925) published in 1925. Volterra (1927) acknowledged Lotka's priority, but stressed that he had formulated principles concerning " "sea-fisheries,', "implying that this was not what Lotka had focused on. Indeed, Lotka derived his version of the Lotka-Volterra model in an entirely different way than Volterra. His approach is in a sense more contemporary than that of Volterra's, pointing towards complex systems theory and its use across the disciplines.

\subsection{The design of the Lotka-Volterra model by Lotka}

In contrast to Vito Volterra, the other author of the Lotka-Volterra model, Alfred Lotka (1880-1949), struggled to gain recognition for his work from the scientific community throughout his life. In addition to being a mathematician and statistician he had a background in physics, physical chemistry, and biology. In his work, Lotka integrated concepts, methods, and techniques from those various fields, developing a modeling approach that could be characterized as a precursor for a systems approach. The developers of general systems theory and cybernetics, Ludwig von Bertalanffy (1968) and Norbert Wiener (1948), were inspired by Lotka's work, especially his book Elements of Physical Biology (1925). Herbert Simon characterized Lotka 'as a forerunner whose imagination creates plans of exploration that he can only partly execute, but who exerts great influence on the work of his successors' (Simon, 1981, p. 493). For Simon, Lotka's book provided insight into how mathe-

\footnotetext{
${ }^{16}$ For example in Volterra (1936) and Volterra (1937a) he discussed the connection between his theories and biological data.

17 The biologists with whom Volterra corresponded included Georgii F. Gause, R. N. Chapman, Jean Régnier, Raymond Pearl, Karl Pearson, D’Arcy W. Thompson, William R. Thompson, Alfred J. Lotka, and Vladimir A. Kostitzin. The correspondence of Vito Volterra on mathematical biology also provides interesting material as regards modeling methods. Among other things, it provides material on the choices between deterministic and probabilistic approaches; between continuous and discrete models; between closed-form solutions and numerical solutions, and between qualitative and quantitative models.
} 
matics could be fruitfully applied in the social sciences. Lotka is also regarded as the founder of mathematical demography, and exerted a great influence on ecologist Eugene P. Odum, who counts as the founder of systems ecology. As the above discussion on Lotka's influence on the development of various systems theoretic approaches already hints at, his design of the Lotka-Volterra model was opposite to that of Volterra. Instead of starting from different simple cases and generalizing from them, he developed a highly abstract and general model template that could be applied to modeling various kinds of systems.

\subsubsection{Physical biology according to Lotka}

Lotka aimed at developing a " 'physical biology,' " employing “ 'physical principles and methods in the contemplation of biological systems" " (Lotka, 1925, p. viii). He was, however, skeptical of applying the most idealized cases of mechanics to biological systems. His main focus was on the evolution of biological systems, which he defined as follows: 'Evolution is the history of a system undergoing irreversible changes' (Lotka, 1925, p. 49). This definition does not exclude reversible processes, although Lotka argued that all real processes are irreversible. Reversible processes were for him idealizations. The evolution of a system in time is characterized, according to Lotka, by an increase in entropy. Physical biology was in turn " 'a branch of the greater discipline of the General Mechanics of Evolution' " (ibid.).

Another important impulse for Lotka's program of physical biology came from the success of physical chemistry by the end of the 19th century (Servos, 1990). Physical chemistry functioned as a model science for Lotka in much the same way as mechanics did for Volterra. Based on the conviction " "that the principles of thermodynamics or of statistical mechanics do actually control the processes occurring in systems in the course of organic evolution" " (Lotka, 1925, p. 39), Lotka set out to apply the methods, techniques, and concepts from thermodynamics and statistical physics to the study of the evolution of biological systems. He realized, however, that biological systems are too complex to allow any straightforward application of thermodynamics. Lotka attempted to overcome this problem by introducing a generalized approach, which can be best understood as a kind of systems approach. The model later dubbed as the Lotka-Volterra model was just one application of Lotka's systems approach.

Apart from mechanics and physical chemistry, also the field of energetics had an impact on Lotka's theorizing. Energetics as a specific theoretical field originated in the 19th century in the works of Georg Helm (1898) and Wilhelm Ostwald (1893) and others. It aimed at the development of a generalized theory based on the concept of energy. In a broader context, the movement can be understood as a reaction against the mechanistic world-view. In addition to being one of the main spokesmen of energetics, Ostwald (1893) was also one of the founding fathers of physical chemistry. From energetics Lotka took the idea of conceptualizing the components 
of systems as energy transformers in an analogy to heat engines (energy transformers could be organisms, chemical elements, etc.).

Energy transformers and the processes linked to them constituted what Lotka called the Micro-Mechanics of a system. Macro-Mechanics on the other hand encompassed the redistribution of mass between the components of the system. This distinction is similar to thermodynamics and statistical mechanics where, according to Lotka, the Macro-Mechanics examines the " "phenomena displayed by the component aggregates in bulk' ", and the Micro-Mechanics " 'is centered primarily upon the phenomena displayed by the individuals of which the aggregates are composed' " (Lotka, 1925, p. 50). Thus Lotka attempted, at the same time, to apply thermodynamics and statistical mechanics to biology and to formulate a general approach that could overcome the problems inherent in drawing direct analogies between different disciplines-as Volterra had done.

\subsubsection{Lotka's systems approach and the Lotka-Volterra model}

In his version of the Lotka-Volterra model, the model concerned macro-level phenomena. In order to describe the general dynamics on the macro level, Lotka started out from the law of mass action used in chemistry to describe the behavior of solutions. Lotka introduced the law by using the example of a system consisting of 4 gram-molecules of hydrogen, 2 gram-molecules of oxygen, and 100 grammolecules of steam, at one atmosphere pressure, and a tempreature of $1800^{\circ} \mathrm{C}$. The equation describing the evolution of this system is of the following form:

$$
\frac{1}{v} \frac{d m_{1}}{d t}=k_{1} \frac{m_{2}^{2} m_{3}}{v^{3}}-k_{2} \frac{m_{1}^{2}}{v^{2}}
$$

where $v$ is the volume, $m_{1}$ is the mass of steam, $m_{2}$ the mass of hydrogen, and $m_{3}$ the mass of oxygen. The coefficients $k_{1}$ and $k_{2}$ are characteristic parameters of the reaction such as temperature and pressure. Lotka was not interested in this particular equation but in the more general statement implied by the equation according to which " the rate of increase in mass, the velocity of growth of one component, steam (mass $m_{1}$ ), is a function of the masses $m_{2}$ and $m_{3}$, as well as of the mass $m_{1}$ itself, and of the parameters $v$ (volume) and $T$ (temperature)' " (Lotka, 1925, p. 42). He then went on to write the equation in a more general form:

$$
\frac{d X_{i}}{d t}=F_{i}\left(X_{1}, X_{2}, . ., . X_{n} ; P, Q\right) .
$$

This equation describes evolution as a process of redistribution of matter among the several components $X_{i}$ of the system. Lotka called this equation the " Fundamental Equation of Kinetics' "where the function $F$ describes the physical interdependence of the several components. $P$ and $Q$ are parameters of the system. $Q$ defines, in the 
case of biological systems, the characters of the species variable in time and $P$ the geometrical constraints of the system such as volume, area, and extension in space.

Lotka had introduced this general approach in two articles published already 5 years before Elements of Physical Biology appeared in print. Interestingly, in both of these articles there appears a pair of equations that has the same form as what Volterra independently arrived at some years later. The first of these was entitled “ 'Analytical note on certain rhythmic relations in organic systems" " (Lotka, 1920a) and the second paper " 'Undamped oscillations derived from the law of mass actions" " (Lotka, 1920b). In the first of the papers, the equations are applied to the analysis of a biological system, and in the second paper they are applied to a chemical system. ${ }^{18}$ The title of the second paper refers explicitly to the law of mass action. In contrast to Volterra, who first considered the simplest models of interaction and then generalized the results to any number of species, Lotka started out from very general considerations and only after he had formulated his general equation did he turn to specific cases, such as the Lotka-Volterra model.

A further, important element in Lotka's design of the Lotka-Volterra model were the methods he introduced to analyze and calculate the dynamic behavior of the systems he had described. Having formulated the fundamental equation of kinetics, Lotka showed that without knowing the precise form of the function describing the interaction between the components, the properties related to the steady states of the system can already be studied. Lotka assumed that both the environment and the genetic constitutions are constant, after which, by means of a Taylor series expansion, he calculated the possible stationary states of the system. He was able to show that, in general, the system will exhibit one of the following three behaviors with increasing time: First, the system asymptotically approaches an equilibrium; second, it performs irregular oscillations around an equilibrium; or third, it performs regular oscillations around the equilibrium. He then applied the fundamental equation to the case of two species, one of which feeds upon the other, arriving at the following equations:

$$
\begin{aligned}
& \frac{d N_{1}}{d t}=\left(\varepsilon_{1}-\gamma_{1} N_{2}\right) N_{1}, \\
& \frac{d N_{2}}{d t}=\left(-\varepsilon_{2}+\gamma_{2} N_{1}\right) N_{2},
\end{aligned}
$$

which are the same as Volterra's equations. They constitute a set of non-linear coupled first-order differential equations, which cannot be solved analytically. Therefore, Lotka's general method of calculating the stationary states became a valuable tool for dealing with such sets of coupled differential equations. As already mentioned, he claimed priority for the model on the basis of his Elements of Physical Biology (1925). The reason for this might be that in (Lotka, 1920a) he draws the Lotka-Volterra equations from his general equation inspired by chemical dynamics

\footnotetext{
${ }^{18}$ Lotka dealt with the rhythmic effects of chemical reactions already in his earlier writings, see e.g. Lotka (1910)
} 
without any discussion of empirical biological systems. In Elements of Physical Biology he applies the equations to the study of a host-parasite system, citing also W. R. Thomson (1922) and L. O. Howard (1897) on this topic. In the third part of the book, the fundamental kinetic equation is also used to study various other cases, such as the spreading of malaria.

As was the case with Volterra, also Lotka's program went much further than the development of what became known as the Lotka-Volterra model. In fact, his visions went far beyond ecology. His book Elements of Physical Biology is a unique conceptualization of the manifold biological, physical, and chemical processes and their complex interactions in the world surrounding us. The organic world becomes a giant energy transformer in which the general kinetic equations provide the mathematical tool for describing the distribution and the transfer of energy between the components of the world. Biological systems, according to Lotka's vision, were to be treated identically with physical systems: it all boiled down to the study of transformations of matter and energy.

\subsection{Discussion}

Lotka and Volterra worked along the same lines, taking inspiration of physical sciences in modeling biological systems, and eventually they presented the same model. Yet they arrived there following different kinds of modeling strategies. While Volterra was making repeatedly use of analogies taken from physical sciences, Lotka was more wary of this kind of procedure and adopted instead a more general, template-based approach. He did not set out to explain some specific dynamics associated with, for example, the spreading of malaria. He focused on more general characteristics of evolving systems, which he defined as follows:

$[\ldots]$ an evolving system is an aggregation of numbered or measured components of several specified kinds, and which observes and registers the history of that system as a record of progressive changes taking place in the distribution, among those components, of the material of which the system is built up. (Lotka, 1925, p. 41)

The template Lotka constructed is a mathematized form of this description of evolving systems. All systems, which show some kind of dynamics, should in principle be describable by means of the template. Such analysis would not be restricted to certain species or populations, but concerned various kinds of transformer types, organic and inorganic.

This difference in the respective modeling strategies of Lotka and Volterra has profound implications concerning the interpretation of their versions of the LotkaVolterra model. Volterra approached modeling from the perspective of the causal explanation of real mechanisms, presenting his model in terms of fully specified equations governing the dynamics of the system in question. This approach enables the ecological interpretation of the coefficients, but simultaneously makes it a gross simplification of the biological reality. Lotka's formulation recognizes the implausibility of completely specifying the full functional forms of the equations governing 
an ecological system, or any other complex system, for that matter. Within a local neighborhood of an equilibrium, the full equations are approximated by the Taylor series expansion (see Haydon and Lloyd (1999, p. 205-206)).

Having elucidated the historical roots of the Lotka-Volterra model, we will now turn to the philosophical discussion on Volterra and modeling. Let us recall that both Weisberg (2007) as well as Scholl and Räz (2013) considered Volterra as a modeler, but on different grounds. Much of what they say on Volterra's theoretical approach is congruent, however, even though they make use of different parts of his work. Weisberg relies exclusively on Volterra's (1926a; 1926b) original publications in Italian and English. Although Scholl and Räz refer to Volterra's early (1926a; 1928) publications, they draw most heavily on the arguments presented in his later work co-authored by D'Ancona (Volterra and D'Ancona, 1935). As we have shown, as Volterra's research program on the biological associations progressed, he started to pay more and more attention to the empirical verification of his models. Thus the somewhat different takes on Volterra's work by Weisberg and Scholl and Räz can be partly explained by their focus on the different phases of his work.

Where Weisberg's and Scholl and Räz's studies part concerns not so much Volterra's actual claims, but rather the more philosophical question of whether Volterra's method of hypothesis can be considered to represent a unique style of theorizing (that Weisberg calls modeling). While Weisberg seems to be working with an already established intuition of modeling that he then chooses to exemplify by contrasting Volterra with Mendeleev and Darwin, the focus of Scholl and Räz is on causal inference. They derive their working notion of modeling as arising from the insufficient epistemic access from the later writings of Volterra (Volterra and D'Ancona 1935) without any explicit attention to the philosophical discussion on modeling. ${ }^{19}$ This differs from Weisberg's treatment. He does, after discussing the different case studies, explicate at length what kind of features " 'an account of models adequate for characterizing the practice of modeling must have' " (Weisberg, 2007, p. 221).

It is noteworthy that apart from agreeing with Weisberg about the indirect nature of Volterra's theoretical endeavour, Scholl and Räz adopt, furthermore, his distinction between representational and dynamical fidelity. What their criticism eventually boils down to, is to showing how Darwin succeeded in what Volterra did not: establishing a " "trajectory from " "how possibly" " (dynamical fidelity without representational fidelity) to " "how actually" (dynamical fidelity and representational fidelity) " "(Scholl and Räz, 2013, p. 131). ${ }^{20}$ For Scholl and Räz, then, modeling

19 Our reading of Scholl and Räz (2013) differs somewhat from their later reading of their own article (this volume). In their original article, they do not clearly offer causal inference as a contrast to modeling (that would provide an alternative for Weisberg's contrast between modeling and abstract direct representation). According to them " "much of our discussion will focus on models of causal structures' " (Scholl and Räz, 2013, p. 117, emphasis added). Their earlier focus was on causal inference in general and modeling a strategy to deal with insufficient epistemic success.

${ }^{20}$ Scholl and Räz adopt the distinction between 'how possibly' and 'how actually' from the discussion on mechanistic explanation (Machamer et al., 2000), a discussion that has been until recently relatively disinterested in modeling and that considered models as explanation sketches only (see Knuuttila and Loettgers (2013)). 
does not need to stay at the level of indirect reasoning and production of only dynamically accurate models, and so indirect representation needs not to be the mark of modeling. Yet Weisberg would not necessarily disagree with them. His thesis concerns the way the model is developed, that is, the indirect strategy of representing a hypothetical system. In that stage, the dynamical fidelity may function as the most important guide. But it does not mean that models could not be developed into representationally more accurate descriptions of actual target systems. That is what Volterra (and D'Ancona) attempted, to some degree. So there seems not to be too big a difference between Weisberg's and Scholl and Räz's claims concerning Volterra's work and modeling.

Scholl and Räz (this volume) seem to be willing to render the contrast between their and Weisberg's account clearer by claiming that Volterra and D'Ancona “ 'write that for their own investigation they would have preferred an experimental approach, which would have allowed for direct causal inferences in the system under scrutiny.' "We find it doubtful that a world-renowned mathematician and theoretical physicist, whose outspoken goal was to mathematize social science and biology would have preferred the experimental approach.

In our view it is more likely that Volterra and D'Ancona's discussion of the rationale of their mathematical approach is due to Volterra's methodological views according to which the empirical verification of theories was important-indicating that Volterra cannot be conceived as a modeller making use of a purposeful strategy of indirect representation. Yet, at retrospect, what Volterra eventually accomplished can be approached as an instance of modeling despite his methodological pronouncements. As we discussed in our case study, Volterra's primary aim, already expressed in his Inaugural Address (Volterra, 1901) was to isolate the " 'fundamental parameters' " of the predator-prey system. In actual practice he started right away from certain assumed factors and from the hypothesis that the oscillations in the fishery data might be accounted for solely by these factors and the resulting interaction of the two species.

From our point of view, the crucial question concerns the reasons as to why Volterra (and D'Ancona) did not achieve more representationally accurate models? Was there something about their method that sets it apart from Darwin's and Mendeleev's achievements? We think that there is a more profound reason for why Volterra adopted an indirect modeling strategy that neither Weisberg, nor Scholl and Räz discuss. Namely, as we showed through our case study, Volterra was interested in the mathematization of biology and social sciences, and in utilizing the tools and methods of mechanics in this task. The way he proceeded to model biological associations made heavy use of analogies to mechanics that enabled him to transfer mathematical tools and methods of physics to biology. The strategy of constructing a simpler hypothetical system to which only some properties are assigned is due to the goal of mathematizing the problem at hand. Such method had proven successful within physics, but Volterra was only too aware of the complexity of the problem posed by biological associations in a natural environment with various kinds of perturbations. The dynamical fidelity was important to Volterra precisely since his was an attempt of explaining the oscillations only by internal causes, i.e. solely by the 
interaction between the two species. A major part of contemporary mathematical modeling across different disciplines is precisely providing these kinds of hypothetical explanations. ${ }^{21}$ In (Knuuttila and Loettgers, 2015) we argue that methodsdrivenness and outcome-orientedness are characteristic features of such modeling exercises.

Yet, as our study concerning the different designs of the Lotka-Volterra model shows, modeling is nevertheless not any unitary theoretical strategy, as Weisberg seems to be claiming. Lotka's strategy for using the model templates ${ }^{22}$ drawn from statistical mechanics and physical chemistry was different from that of Volterra's. For Lotka the general systems approach he developed provided the justification for his modeling approach. As we have discussed, his approach anticipates many contemporary modeling practices in which the model templates developed in complex systems theory are applied across the variety of disciplines, studying both natural and social systems. Interestingly, in the context of the study of complex systems, the Lotka-Volterra model was " "rediscovered" " as one of the basic simple models that afforded the study of complex systems (e.g. May (1974)).

\subsection{Conclusion: Theoretical case-based philosophical practice}

How do philosophers of science make use of historical case studies? In particular, are their accounts of historical cases necessarily purpose-built and lacking in evidential strength as a result of putting forth and discussing philosophical positions? In order to find this out, we have examined three different philosophical case studies on Vito Volterra's work, one of which is our own. The interesting outcome of this exercise is that while the philosophical conclusions of the three case studies are different, they largely agree on their interpretation of Volterra's work! The comparison of these case studies discussed (by Weisberg (2007), Scholl and Räz (2013), and Knuuttila and Loettgers (2015)) does not lend credence to the idea that they argue for their philosophical claims by simply confronting them with historical cases - or constructing the cases according to their preferred theory (cf. Pitt (2001)). Does this mean that historical case studies in philosophy of science should be understood as interpretative activity investigating scientific concepts, norms and practices, as Schickore has suggested? Our answer to this question is positive, but we do not see that it would need to imply either rejecting the evidential role of case studies (cf. Schickore (2011)), or compromising, or lessening, their evidential value (cf. Kinzel (2015)). In our view, philosophers of science usually use case studies as vehicles of theoretical reflection, as resources in examining, questioning, and devel-

\footnotetext{
21 The " 'how-possible" " may be a bit misleading expression in this context, since what Volterra accomplished was an alternative explanation for the prevailing explanations that attributed the fluctuations to external causes.

${ }^{22}$ A model template is an abstract conceptual idea concerning usually a certain kind of interaction and associated with particular mathematical forms and computational methods, see for further discussion Knuuttila and Loettgers (2014).
} 
oping philosophical concepts and accounts. In that use evidential and hermeneutic roles go hand in hand, informing each other. Thus the three case studies presented in this paper serve as examples of case-based theoretical philosophical practice that is underlined by the way each of them uses the strategy of contrasting partially similar, and partially different scientific examples. The use of the contrasting example highlights the conceptual distinctions made.

How should one, then, understand the philosophical case-based theoretical practice? The first thing to notice is that it is difficult to recognize it, if one approaches philosophy as an activity that aims only at a general/rational reconstruction of scientific activity (although that would also need historical and empirical knowledge, if only to recognize what constitutes, in fact, successful science). It seems that this kind of conception of the philosophy of science lies behind the various iterations of the claims that scientific case studies cannot give evidential support for philosophical positions. But clearly, philosophical theorizing also contains an important descriptive component as well as being often more local and tentative in nature - as the practice-oriented philosophy of science has recently shown.

If one looks at the use of historical case studies as vehicles for philosophical theorizing, nothing very special seems to be going on there. Also in scientific research using case studies as a resource for investigation one has to negotiate the relationship between the generalizable insights and the context-specific details. Even in the physical sciences single cases and experiments are sufficient for theorizing, as ShraderFrechette and McCoy (1994) point out in their study of case-based reasoning within ecological sciences. According to them, ecologists often prefer case-specific knowledge coupled with conceptual and methodological analysis to " "ecological theorizing based on untestable principles and deductive inferences drawn from mathematical models' "(p. 244). The case-specific local knowledge allows various kinds of inferences: they can be local to local, or local to many. In local to many reasoning, the local knowledge is desituated to a more generic level, or used to construct typical representatives or exemplars (see Morgan (2014)).

What needs to be recognized is that most scientific knowledge is local, or constructed from the local knowledge, being subject to various initial conditions and environmental contexts. Moreover, while case studies provide a springboard for theorizing and generalization, they are often also used to question earlier held theoretical views, or their generality. The case study methodology has also advantages that spring forth from the way the evidential is woven together with theoretical. A historical case study typically presents " 'a complex, often narrated, account that ... contains some of the raw evidence as well as its analysis and that ties together many different bits and pieces in the study" " (Morgan, 2012, p. 668). Thus narrative becomes a way to deal with what Morgan calls " 'evidential density,', which contributes to theoretical development by offering rich resources for a critical study of different theoretical perspectives. This evidential richness is clearly one of the benefits of case studies: many relevant factors do not need to be abstracted away or shielded, as with laboratory studies and mathematical modeling. Consequently, it seems a mistake to try to tease the evidential dimension of case studies apart from their conceptual and interpretative content. Both are woven together in the theoret- 
ical narrative that aims to integrate different kinds and bits of evidence by showing their interdependence (Morgan, 2012, p. 675). This theoretical-cum-conceptual modality of case studies is so strong that even when case studies succeed to identify a novel interesting phenomenon, like the 'street corner society' (Whyte, 1943), 'the community's response was to understand the phenomena revealed as potentially generic' (Morgan, 2012, p. 673).

It is our claim, then, that the key to the epistemic value of case studies, in philosophy of science, like in both natural and social sciences, lies in the way they weave together different kinds of evidence with the conceptual analysis and theoretical development. The observation that the same cases may be interpreted differently does not seem to us so grave an objection, since, as pointed out by numerous scholars, case studies often breed new interpretations of the same cases, as well as attempts to confirm the results by new case studies and independent data (e.g. Shrader-Frechette and McCoy (1994)). The three cases provide a good example of this practice of presenting related case studies. As we have argued, they are largely in agreement concerning Volterra's work, ${ }^{23}$ and the theoretical and interpretative element can most clearly be located to how Volterra's work is contrasted with the work of other scholars: Mendeleev, Darwin, and Lotka.

Finally, we remain skeptical of the idea that to justify case study methodology, the cases should be typical of their kind-how do we know the typical without any cases?-or somehow important or critical. The scientific record does not seem to lend support to these kinds of claims either. Ankeny (2012) argues concerning developmental biology that many model organisms used as kind of 'cases' are now regarded as presenting typical patterns of phenomena, while they were often originally selected for study for other reasons, such as convenience or ease of experimental manipulation. And even when they turned out atypical, they still continued to provide a focal point in the field, permitting investigation of variations in phenomena or processes. We see this kind phenomenon taking shape also with the three case studies on Volterra, each of which presents variations on the theme of modeling, delineated with the help of contrasting Volterra's work with that of other theorists.

\section{References}

Ankeny, R. A. (2012). Detecting themes and variations: The use of cases in developmental biology. Philosophy of Science, 79:644-654.

Bailer-Jones, D. (2009). Scientific Models in Philosophy of Science. Pittsburgh: University Press.

Burian, R. (2001). The dilemma of case studies resolved: The virtues of using case studies in the history and philosophy of science. Perspectives on Science, 9:383404.

\footnotetext{
${ }^{23}$ Even though the differences between the three case studies with respect to Volterra's work were more substantial, such underdetermination of theories by data would be a common feature of other scientific practices, too.
} 
Buss, L. (1987). The Evolution of Individuality. Princeton University Press.

Chang, H. (2012). Is Water $\mathrm{H}_{2} \mathrm{O}$ ? Evidence, Pluralism and Realism. Boston Studies in the Philosophy and History of Science. Dordrecht:Springer.

Contessa, G. (2007). Scientific representation, interpretation, and surrogative reasoning. Philosophy of Science, 74:48-68.

da Costa, N. C. A. and French, S. (2000). Models, theories and structures: Thirty years on. Philosophy of Science, 67:116-127.

Darwin, C. (1882). The Origin of the Species by Means of Natural Selection. Murray.

French, S. and Ladyman, J. (1999). Reinflating the semantic approach. International Studies in the Philosophy of Science, 13:103-21.

Frigg, R. (2010). Models and fiction. Synthese, 172:251-268.

Gause, G. (1935). Studies in the ecology of the orthoptera. Ecology, 11:307-325.

Giere, R. N. (2004). How models are used to represent reality. Philosophy of Science (Symposia), 71:742-752.

Godfrey-Smith, P. (2006). The strategy of model-based science. Biology and Philosophy, 21:725-740.

Haydon, D. and Lloyd, A. (1999). On the origins of the Lotka-Volterra equations. Bulletin of the Ecological Society of America, 80:205-206.

Helm, G. (1898). Die Energetik. Verlag von Veit.

Howard, L. O. (1897). A study in insect parasitism: A consideration of the parasites of the white-marked tussok moth with an account of their habits and interrelations, and with descriptions of new species. U.S. Dep. Agric. Tech. Bull, 5:1-57.

Humphreys, P. (2002). Computational models. Proceedings of the Philosophy of Science Association, 3:S1-S11.

Humphreys, P. (2004). Extending Ourselves: Computational Science, Empiricism, and Scientific Method. Oxford University Press.

Israel, G. (1991). Volterra's analytical mechanics of biological associations (second part). U.S. Dep. Agric. Tech. Bull., 5:1-57.

Israel, G. (1993). The emergence of biomathematics and the case of population dynamics: A revival of mechanical reductionism and darwinism. Science in Context, 6:469-509.

Israel, G. and Gasca, A. M. (2002). The Biology of Numbers: The Correspondence of Vito Volterra on Mathematical Biology. Birkhäuser.

Kinzel, K. (2015). Narrative and evidence: How can case-studies from the history of science support claims in the philosophy of science? Studies in History and Philosophy of Science Part A, 49:48-57.

Knuuttila, T. (2005). Models, representation, and mediator. Philosophy of Science, $72: 1260-1271$.

Knuuttila, T. (2006). From representation to production: Parsers and parsing in language technology. Sociology of Science, 25:41-55.

Knuuttila, T. (2013). Science in a new mode: Good old (theoretical) science vs. brave new (commodified) knowledge production. Science and Education, 22:2443-2461. 
Knuuttila, T. and Loettgers, A. (2013). The productive tension: Mechanisms vs. templates in modeling the phenomena. In Humphreys, P. and Imbert, C., editors, Representations, Models, and Simulations, pages 3-24. Routledge.

Knuuttila, T. and Loettgers, A. (2014). Varieties of noise: Analogical reasoning in synthetic biology. Studies in History and Philosophy of Science Part A, 48:76-88.

Knuuttila, T. and Loettgers, A. (2015). Modelling as indirect representation? the Lotka-Volterra model revisited. The British Journal for the Philosophy of Science, in press.

Lancaster, E. R. (1884). The scientific results of the exhibition. Fisheries Exhibition Literature, 4:405-442.

Loettgers, A. (2007). Model organisms, mathematical models, and synthetic models in exploring gene regulatory mechanisms. Biological Theory, 2:134-142.

Lotka, A. (1910). Contribution to the theory of periodic reactions. The Journal of Physical Chemistry, 14:271-274.

Lotka, A. (1920a). Analytical note on certain rhythmic relations in organic systems. Proceedings of the National Academy of Art and Science, 42:410-415.

Lotka, A. (1920b). Undamped oscillations derived from the law of mass action. Journal of the American Chemical Society, 42:1595-1598.

Lotka, A. (1925). Elements of Physical Biolgy. Williams and Wilkins.

Machamer, P. K., Darden, L., and Craver, C. (2000). Thinking about mechanisms. Philosophie of Science, 67:1-25.

Maeki, U. (2009). Missing the world: Models as isolations and credible surrogate systems. Erkenntnis, 70:29-43.

May, R. (1974). Biological populations with nonoverlapping genertations: Stable cycles, and chaos. Science, 186:645-647.

Maynard-Smith, J. and E. Smathmáry, J. (1995). The Major Transitions in Evolution. Oxford University Press.

Morgan, M. (2012). Case studies: One observation or many? justification or discovery? Philosophy of Science, 79:667-677.

Morgan, M. (2014). Resulting knowledge: Generic strategies and case studies. Philosophy of Science, 81:1012-1024.

Morgan, M. and Morrison, M. (1999). Models as mediating instruments. In Morgan, M. and Morrison, M., editors, Models as Mediators. Perspectives on Natural and Social Science, pages 97-146. Cambridge University Press.

Ostwald, W. (1893). Lehrbuch der allgemeinen Chemie: Chemische Energie. Wilhelm Engelman.

Pitt, J. C. (2001). The dilemma of case studies: Toward a heraclitian philosophy of science. Perspectives on Science, 9:373-382.

Schickore, J. (2011). More thoughts on HPS: Another 20 years later. Perspectives on Science, 19:453-481.

Scholl, R. and Räz, T. (2013). Modeling causal structures: Volterra's struggle and Darwin's success. European Journal for Philosophy of Science, 3:115-132.

Scudo, F. (1971). Vito Volterra and theoretical ecology. Theoretical Population Biology, 2:1-23. 
Scudo, F. (1992). Vito Volterra, ecology and the quantification of Darwinism. Atti del Convegno internazionale in memorie di Vito Volterra, Rome. Accademia Nazionale dei Lincei.

Scudo, F. M. and Ziegler, J. R. (1978). In The Golden Age of Theoretical Ecology: 1923-1940. Springer-Verlag.

Servos, J. W. (1990). Physical Chemistry from Ostwald to Pauling: The Making of a Science in America. Princeton University Press.

Shrader-Frechette, K. S. and McCoy, E. D. (1994). How the tail wags the dog: How value judgments determine ecological science. Environmental Values, 3:107-120.

Simon, H. (1981). The Sciences of the Artifical. MIT Press.

Suárez, M. (2008). Scientific fictions as rules of inference. In Suárez, M., editor, Fictions in science: philosophical essays on modeling and idealisation, pages 258-270. Routledge.

Thomson, W. R. (1922). Théorie de l'action des parasites entomophages. les formules mathématiques du parasitisme cyclique. Comptes Rendus Acad. Sci. Paris, 174:1202-1204.

Volterra, V. (1901). On the attempts to apply mathematics to the biological and social sciences. In Goodstein, J. D., editor, The Volterra Chronicles: Life and Times of an Extraordinary Mathematician 1860-1940, pages 247-260. AMS.

Volterra, V. (1926a). Fluctuations in the abundance of a species considered mathematically. Nature, CXVIII:558-560.

Volterra, V. (1926b). Variazioni e fluttuazioni del numero d'indivui in specie animali conviventi. Memorie della R. Accademia Lincei, 2:31-113.

Volterra, V. (1927). Letter to nature. Nature, 119:12.

Volterra, V. (1928). Variations and fluctuations of the number of individuals in animal species living together. Journal du Conseil International Pour l'Exploration de la Mer, 3:3-51.

Volterra, V. (1930). Theory of Functionals and of Integral and Integro-Differential Equations. Blackie and Son.

Volterra, V. (1931). Leçons sur la Théorie Mathématique de la Lutte Pour la Vie. Gauthier-Villars.

Volterra, V. (1936). La théorie mathématique de la lutte pour la vie et l'expérience (a propos de deux ouvrages de C.F. Gause). Scientia, LX:169-174.

Volterra, V. (1937a). Applications des mathématiques á la biologie'. L'Enseignement Mathématique, XXXVI:297-330.

Volterra, V. (1937b). Principes de biologie mathématique. Acta Biotheoretica, III:136.

Volterra, V. and D'Ancona, U. (1935). Les associations biologiques au point de vue mathématique. Hermann.

von Bertalanffy, L. (1968). General System Theory: Foundations, Development, Applications. George Braziller.

Weisberg, M. (2007). Who is a modeler? British Journal for the Philosophy of Science, 58(2):207-233.

Weisberg, M. (2013). Simulation and Similarity: Using Models to Understand the World. Oxford University Press. 
Weisberg, M. and Reisman, K. (2008). The robust Volterra principle. Philosophy of Science, 75:106-131.

Whyte, F. (1943). The Street Corner Society. The Social Structure of an Italian Slum. The University of Chicago Press.

Wiener, N. (1948). Cybernetics or Control and Communication in the Animal and the Machine. MIT Press. 